\title{
Multi-player race
}

\author{
Serhat Doğan a , Emin Karagözoğlu ${ }^{\mathrm{a}, \mathrm{b}}$, Kerim Keskin ${ }^{\mathrm{c}, *}$, Çağrı Sağlam ${ }^{\mathrm{a}}$ \\ a Department of Economics, Bilkent University, Ankara 06800, Turkey \\ ${ }^{\mathrm{b}}$ CESifo, Poschingerstr. 5, Munich 81679, Germany \\ ' Department of Economics, Kadir Has University, Istanbul 34083, Turkey
}

\section{A R T I C L E I N F O}

\section{Article history:}

Received 20 November 2017

Revised 8 March 2018

Accepted 10 March 2018

JEL classification:

C72

D72

D74

\section{Keywords:}

Contests

Discouragement effect

Dynamic games

Momentum effect

Race

\begin{abstract}
A B S T R A C T
We present a model of race with multiple players and study players' effort choices and expected prizes in equilibrium. We show that, in equilibrium, once any two players win one battle each, the remaining players do not exert any effort anymore. This turns the continuation game into a two-player race. This is different than the results in previous two-player models of race, which report that all states of the game are reached with positive probabilities. We also provide a set of comparative static results on the effects of the number of players and the victory threshold.
\end{abstract}

(c) 2018 Elsevier B.V. All rights reserved.

\section{Introduction}

Multi-battle competitive interactions are ubiquitous. Some obvious examples are sports tournaments, patent races, election campaigns, public debates, wars, etc. The contest theory literature produced various models of multi-battle dynamic contests, such as race, tug-of-war, elimination tournaments, war of attrition, and repeated incumbency fights (see Konrad, 2012; Vojnovic, 2016). This paper studies a model of race. For almost all examples mentioned above, it would be natural to consider a multi-player interaction. Interestingly, earlier work on race exclusively focused on two-player interactions. Here, we study a multi-player race.

In a race, ${ }^{1}$ players simultaneously decide on the efforts they exert in each battle and the player who first accumulates a certain number of battle victories wins the contest. We study a multi-player game of race, where (i) the battle outcomes are determined with a Tullock contest success function (CSF), (ii) there is no intermediate prize, (iii) no losing punishment, and (iv) no discounting. Players are symmetric in that they have identical winning prizes and cost functions, and the victory threshold (i.e., the number of victories needed to win the contest) is the same for each player. We describe the differences and similarities between our model and the earlier work on race in detail, in Section 2.

\footnotetext{
* Corresponding author.

E-mail address: kerim.keskin@khas.edu.tr (K. Keskin).

1 We adopt the definition put forward by Konrad and Kovenock (2009).
} 
Table 1

Some recent studies on two-player races and Colonel Blotto games.

\begin{tabular}{lll}
\hline & Tullock CSF & All-pay CSF \\
\hline Dynamic Models & Theoretical: & Theoretical: \\
& Baba (2012); & Gelder (2014); \\
& Harris and Vickers (1987); & Konrad and Kovenock (2009) \\
& Klumpp and Polborn (2006) \\
& Experimental: & Experimental: \\
& Baba (2012); & Deck and Sheremeta (2012); \\
& Mago et al. (2013); & Mago and Sheremeta (2017) \\
& Irfanoglu et al. (2015) & \\
& Theoretical: & \\
& Klumpp and Polborn (2006); & Theoretical: \\
Robson (2005) & Hart (2008); \\
& Experimental: & Kvasov (2007); \\
& Chowdhury et al. (2013); & Roberson (2006) \\
& Duffy and Matros (2017); & Experimental: \\
& Irfanoglu et al. (2015) & Chowdhury et al. (2013); \\
& & Mago and Sheremeta (2017) \\
\hline
\end{tabular}

We show that, in equilibrium, once any two players win one battle each, all of the other players are totally discouraged, i.e., they do not exert any effort in the continuation game, which turns the continuation game into a (symmetric or asymmetric) two-player race. This finding is different than the earlier theoretical works on two-player race (e.g., Gelder, 2014; Klumpp and Polborn, 2006; Konrad and Kovenock, 2009), which documents pervasiveness (i.e., all states of the game are reached with positive probabilities).

This result shares a flavor similar to the well-known Duverger's Law in the theory of elections, which states that plurality voting tends to favor a two-party system (Duverger, 1972). Race is similar to plurality voting in that it does not impose any requirement on the winning margin but only imposes an absolute (or, nominal) victory threshold. We show that race produces a two-player contest on the equilibrium path, which can be read as a strategic foundation for the contest theory version of the Duverger's Law. In addition to that, if one entertains an application of our model to elections (as in Klumpp and Polborn, 2006), our main result could also be interpreted as a strategic support for elections with runoffs, where only two candidates qualify for the second round.

We also conduct comparative static analyses on the number of players and the victory threshold to see their effect on important markers of dynamic contests such as the discouragement effect (decrease in the efforts of laggards), rent dissipation (the ratio of exerted effort to the prize value), and the momentum effect (increased chances of the player who won the first battle for winning the second battle or the whole contest). The results can be summarized as follows: (i) The aforementioned extreme form of discouragement is present independent of the number of players as long as this number is greater than two. (ii) In a model with a victory threshold of two, an increase in the number of players increases rent dissipation and tempers the momentum effect. (iii) Interestingly, in a model with three players, an increase in the victory threshold first decreases then increases rent dissipation. The converse is true for the momentum effect. A victory threshold of four minimizes rent dissipation and maximizes the momentum effect.

The organization of the paper is as follows. In Section 2, we present an overview of the related literature. In Section 3, we first present our results for the case of three-player race $(n=3)$ with a victory threshold of two $(t=2)$. Later, we extend our results to the models with more than three players (keeping $t=2$ ) and with a victory threshold of more than two (keeping $n=3$ ). Section 4 concludes.

\section{Literature review}

The literature on dynamic contests is large (see Konrad, 2012; Vojnovic, 2016). Here, we primarily focus on earlier works on race. The first paper to formally study race as a dynamic multi-stage contest (with simultaneous actions in each stage) is Harris and Vickers (1987). ${ }^{2}$ These authors model a patent race between two firms as a multi-stage contest with sequential battles. In each stage (or, battle), firms simultaneously spend efforts/resources in research and development; and the battle outcome is a stochastic function of their efforts. The first firm to reach a certain number of stage victories wins the patent race. The authors show that the leader spends greater effort than the follower does, and these efforts increase as the gap between the two firms decreases.

Our paper is most closely related to Klumpp and Polborn (2006), who study the primaries, i.e., sequential elections in single states in the U.S., as a two-player race. The authors compare two alternative temporal structures (dynamic vs. static) on the basis of the prevalence of the momentum effect, the expected costs, and the candidates' winning probabilities. Using a Tullock CSF to determine the outcome of individual elections, they show that players spend relatively large amounts of resources in the first battle and that winning the first battle creates a momentum. The discouragement is not extreme in the sense that the laggard keeps investing non-zero amounts.

\footnotetext{
${ }^{2}$ Harris and Vickers (1985) also study a race, but in their model players take actions sequentially, which does not fit the definition we adopt here.
} 


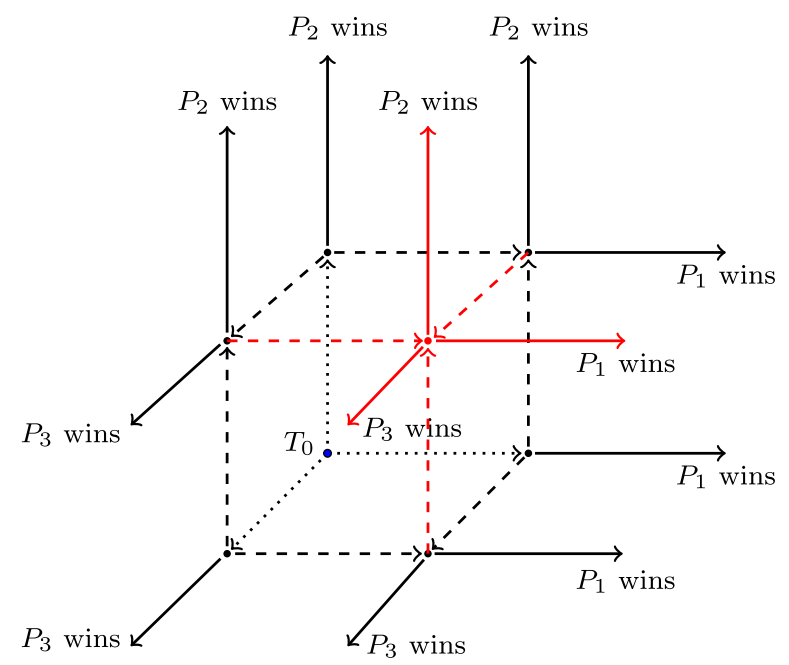

Fig. 1. Race with $n=3$ and $t=2$.

Konrad and Kovenock (2009) also study a dynamic two-player race. Differently from Klumpp and Polborn (2006), these authors utilize an all-pay auction CSF and allow for intermediate prizes. They characterize the unique subgame perfect Nash equilibrium, which is in mixed strategies, and show that even when one player leads by a large margin, the laggard does not give up; and consequently, each state in the game is reached with a positive probability.

More recently, Gelder (2014) introduces losing penalties and discounting into a two-player race. The author utilizes an all-pay auction CSF as in Konrad and Kovenock (2009); and he investigates the last stand behavior as well as the likelihood that the laggard will catch up. He shows that losing penalties (especially when large relative to the winning prize) and discounting make neck-to-neck races more likely and temper the momentum effect.

It is worthwhile mentioning that the Colonel Blotto game (see Borel, 1921) can be thought of as a static version of race with resource constraints. ${ }^{3}$ With this interpretation in mind, our paper is also related to some earlier works on Colonel Blotto games. For recent theoretical papers studying two-player Colonel Blotto games, the reader is referred to Hart (2008); Kvasov (2007); Roberson (2006); Robson (2005), and Baba (2012) among others. Especially in recent years, the experimental literature on races and Colonel Blotto games have been very productive. In particular, Deck and Sheremeta (2012) examine attacking and defending strategies in the game of siege; Baba (2012) analyze individual behavior in simultaneous and sequential Colonel Blotto games; Chowdhury et al. (2013) investigate Colonel Blotto games with asymmetric resources; Mago et al. (2013) examine the influence of luck and intermediate prizes on effort choices in a Tullock race; Irfanoglu et al. (2015) and Mago and Sheremeta (2017) analyze individual behavior in static and dynamic multi-battle contests; and Duffy and Matros (2017) consider asymmetric values and different payoff objectives in stochastic Colonel Blotto games.

Table 1 categorizes earlier works mentioned above based on three dimensions: (i) temporality (simultaneous vs. sequential), (ii) CSF used (Tullock vs. all-pay), and (iii) methodology (theoretical vs. experimental).

Our paper theoretically investigates a dynamic race using a Tullock CSF. Arguably, the most important difference between our paper and all papers mentioned above is that we study a multi-player race. This places our model in a fourth dimension outside Table 1. Additionally, we differ from the other existing theoretical work with dynamic models, by assuming no intermediate prize (differently from Konrad and Kovenock, 2009) and by assuming no losing penalty or discounting (differently from Gelder, 2014).

\section{Model and results}

\subsection{Race with $n=3$ and $t=2$}

The set of players is denoted by $N=\{1,2,3\}$. Players compete in the game shown in Fig. 1, where each player $i \in N$ chooses a contest effort $e_{i}^{t} \in[0, \infty)$ at each node $t$ which corresponds to a specific battle. ${ }^{4}$ Player $i$ 's probability of winning

\footnotetext{
${ }^{3}$ We would like to thank the reviewer for bringing this relationship to our attention.

${ }^{4}$ The game tree is, in fact, more detailed with infinitely many branches (representing every possible effort level) coming out of each node for each player. Since the players' actions depend only on the current state, the game can be summarized as in Fig. 1.
} 
the battle at $t$ is determined by the following contest success function (see Tullock, 1980):

$$
p_{i}\left(e_{1}^{t}, e_{2}^{t}, e_{3}^{t}\right)=\frac{e_{i}^{t}}{e_{1}^{t}+e_{2}^{t}+e_{3}^{t}} .
$$

The cost function is linear as in Klumpp and Polborn (2006); Konrad and Kovenock (2009), and Gelder (2014):

$$
c_{i}(e)=k e
$$

for some $k>0$ and every $i \in N$.

Players start at $T_{0} \equiv(0,0,0)$ and $(i)$ a win by Player 1 results in a move along the $x$-axis; (ii) a win by Player 2 results in a move along the $y$-axis; and (iii) a win by Player 3 results in a move along the $z$-axis. Player $i$ wins the race if he wins two victories before any of the other players does. The player who wins the race receives a prize denoted by $V>0$. All the remaining players receive a normalized prize of 0 . There are no intermediate prizes and players do not discount future. We denote this game with three players and a victory threshold of two by $\Gamma^{3,2}$.

As a concrete example, consider three researchers (or research groups) working on independent projects on the same research question. Each researcher's aim is to complete his project and publish an article before one of the other researchers does. For the sake of simplicity, assume that for a given research question, only the first published article makes an impact. Hence, if one researcher publishes before the others, the research efforts already exerted by the other researchers go for nothing. Similar to R\&D or patent races, this strategic interaction can be modeled as a multi-player race (as in $\Gamma^{3,2}$ ). The first battle victory would correspond to having an online-available working paper, and the second battle victory would correspond to have the paper published as an article. We will revisit this example when we interpret our results below.

Throughout the paper, the equilibrium concept used is (pure strategy) subgame perfect Nash equilibrium. Given that previously exerted efforts are sunk costs, a player's optimal strategy at a given node is independent of the previous effort levels. Hence, focusing on state-dependent strategies does not cause any loss of generality.

In Proposition 1, we characterize the equilibrium efforts and expected prizes in $\Gamma^{3,2}$. Before doing that, we need to introduce some more notations: Let $T_{3}$ denote the node on which all three players have one victory each. Thus, $T_{3} \equiv(1,1$, 1). Similarly, $T_{2} \in\{(1,1,0),(1,0,1),(0,1,1)\}$ and $T_{1} \in\{(1,0,0),(0,1,0),(0,0,1)\}$. As mentioned above, $T_{0} \equiv(0,0,0)$ is the beginning of the game, where no player has any victory yet. In the proposition below, symmetries in players' cost functions and starting positions allow us to focus - without loss of generality- on one of the possible nodes for $T_{1}$ and $T_{2}$. Hence, with a slight abuse of notation, we take $T_{2}=(1,1,0)$ and $T_{1}=(1,0,0)$ below. Finally, let $e_{i}^{T_{m}}$ denote player $i$ 's effort at node $T_{m}$ for every $i \in N$ and every $m=0,1,2,3$.

Proposition 1. The subgame perfect Nash equilibrium of $\Gamma^{3,2}$ is characterized by

$$
\begin{aligned}
e^{T_{3} *} & =\left(\frac{2 V}{9 k}, \frac{2 V}{9 k}, \frac{2 V}{9 k}\right) & & \text { at } T_{3}=(1,1,1) ; \\
e^{T_{2} *} & =\left(\frac{V}{4 k}, \frac{V}{4 k}, 0\right) & & \text { at } T_{2}=(1,1,0) ; \\
e^{T_{1} *} & =\left(\frac{15 V}{98 k}, \frac{3 V}{98 k}, \frac{3 V}{98 k}\right) & & \text { at } T_{1}=(1,0,0) ; \text { and } \\
e^{T_{0} *} & =\left(\frac{41 V}{294 k}, \frac{41 V}{294 k}, \frac{41 V}{294 k}\right) & & \text { at } T_{0}=(0,0,0) .
\end{aligned}
$$

Moreover, the equilibrium expected prizes are

$$
E V^{T_{0} *}=\left(\frac{22 V}{294}, \frac{22 V}{294}, \frac{22 V}{294}\right) \text {. }
$$

Proof. See the Appendix.

Note that the equilibrium reported in Proposition 1 is unique. It is also worthwhile emphasizing that the equilibrium concept we employ allows us to derive equilibrium efforts in all nodes, on and off the equilibrium path. Hence, even though the players in our model start the race at a neutral state (i.e., $T_{0}$ ), our equilibrium characterization captures all other cases in which the players start the race at some non-neutral state. This observation will still be valid later, when we extend our analysis to a race with $n>3$ players and/or a victory threshold of $t>2$.

Below, we compare the equilibrium of $\Gamma^{3,2}$ with the equilibrium outcomes of the two-player race in Klumpp and Polborn (2006) along three dimensions: the discouragement effect, rent dissipation, and the momentum effect.

Discouragement effect: At the node $T_{2}=(1,1,0)$, i.e., after two different players win one battle each, the player who fell behind becomes totally discouraged exerting no contest effort. This is an extreme case of the well-known discouragement effect (see Konrad, 2012). An important implication of Proposition 1 is that the three-player race reduces to the two-player race at $T_{2}$.

The intuition for this result is as follows: at $T_{1}$, there are two laggards, and their expected prizes at this particular node makes it still worthwhile exerting positive effort for each one of them. However, at $T_{2}$, the competition between the two leaders (i.e., the players with one victory each) makes them even more aggressive (e.g., the sum of their efforts at $T_{2}$ is 
larger than two times the effort of the leader at $T_{1}$ ), pushing down the expected prize of the laggard to such low values that it is not worthwhile for him to exert a positive effort anymore.

Recall the aforementioned "publication contest" example. If researcher $A$ makes a working paper available online, then researchers $B$ and $C$ would be discouraged and decrease their research efforts, but they would not completely give up on their own projects. Now, suppose without loss of generality that $B$ manages to release a working paper before $A$ could publish his. In that case, $A$ and $B$ would try harder now, to outperform each other; and this intense competition between them, which reveals itself as increased efforts by both, makes $C$ become totally discouraged. ${ }^{5}$

Rent dissipation: The rent is more dissipated in $\Gamma^{3,2}$ compared to the two-player race. Normalizing the winning prize and the marginal cost of effort to 1, Klumpp and Polborn (2006) show that at the node $(0,0)$, both players exert 21/128, producing a total effort of 0.328 . At the node $(1,0)$, they exert $9 / 64$ and $3 / 64$, respectively, producing a total effort of 0.188 . At the node $(1,1)$, which is to be reached with probability $1 / 4$, both players exert $1 / 4$, producing a total effort of 0.5 (in expectation, 0.125$)$. Hence, the total rent dissipation turns out to be 0.6406 . In our three-player race, at the node $(0,0,0)$, all players exert $41 \mathrm{~V} / 294 k$, producing a total effort of $0.418 \mathrm{~V} / \mathrm{k}$. At the node $(1,0,0)$, they exert $15 \mathrm{~V} / 98 \mathrm{k}, 3 \mathrm{~V} / 98 \mathrm{k}$, and $3 \mathrm{~V} / 98 \mathrm{k}$, respectively, producing a total effort of $0.214 \mathrm{~V} / \mathrm{k}$. At the node $(1,1,0)$, which is to be reached with probability $2 / 7$, both of the leading players exert $V / 4 k$, producing a total effort of $0.5 \mathrm{~V} / \mathrm{k}$ (in expectation, $0.143 \mathrm{~V} / \mathrm{k}$ ). Hence, the total rent dissipation is 0.7755 , which is larger than 0.6406 of the two-player race.

The intuition for this result is relatively straightforward: a three-player race is more competitive than a two-player race. Thus, players exert higher total efforts in a three-player race. As a matter of fact, in Section 3.2 we show for $t=2$ that as $n$ increases, the total rent dissipation monotonically increases and there is complete rent dissipation in the limit.

Momentum effect: In $\Gamma^{3,2}$, the momentum effect (defined in terms of winning probabilities) is less pronounced compared to the two-player race. More precisely, in Klumpp and Polborn (2006), the winner of the first battle wins the second one with probability $3 / 4$ and wins the whole contest with probability $7 / 8$. In $\Gamma^{3,2}$, the winner of the first battle wins the second one with probability 5/7 and wins the whole contest with probability 6/7.

In the "publication contest" example, the researcher who first made the working paper available online will have an advantage in the following periods, in the sense that he has very high chances of publishing the paper before the other researchers do. However, compared to a two-player race, this advantage is weaker in $\Gamma^{3,2}$, since after a first period victory, there are now two laggards who still try to win the race. Finally, it is worth noting that the momentum effect being weaker compared to a two-player race is compatible with the intense competition at $T_{2}$ in $\Gamma^{3,2}$ mentioned above.

\subsection{Extension to $\mathrm{n}>3$ and $t=2$}

Assume that $N=\{1,2,3, \ldots, n\}$. Each player tries to win $t=2$ battles before any of the other players does. Let $T_{m}$ denote a node on which $m \leq n$ players have one victory each and the other players have no victory. Surely, $T_{m}$ is set-valued for any $0<m<n$. As we did in Proposition 1, in the proposition below -without loss of generality- we take $T_{m} \equiv(1, \ldots, 1,0, \ldots, 0)$ for any $0<m<n$.

Proposition 2. The subgame perfect Nash equilibrium of $\Gamma^{n, 2}$ is characterized by

$$
\begin{aligned}
e^{T_{n} *} & =\left(\frac{(n-1)}{n^{2}} \frac{V}{k}, \ldots, \frac{(n-1)}{n^{2}} \frac{V}{k}\right) & & \text { at } T_{n}, \\
e^{T_{m} *} & =\left(\frac{(m-1)}{m^{2}} \frac{V}{k}, \ldots, \frac{(m-1)}{m^{2}} \frac{V}{k}, 0, \ldots, 0\right) & & \text { at } T_{m} \quad \text { for } 1<m<n, \\
e_{1}^{T_{1} *} & =(2 n-1) \frac{3 n-3}{4(3 n-2)^{2}} \frac{V}{k} \text { and } \quad e_{i}^{T_{1} *}=\frac{3 n-3}{4(3 n-2)^{2}} \frac{V}{k} & \forall i \in N \backslash\{1\} & \text { at } T_{1},
\end{aligned}
$$

Moreover, the equilibrium expected prizes are

$$
E V^{T_{0} *}=\left(\frac{11 n^{2}-12 n+3}{2 n^{2}(3 n-2)^{2}} V, \ldots, \frac{11 n^{2}-12 n+3}{2 n^{2}(3 n-2)^{2}} V\right)
$$

Proof. See the Appendix.

Proposition 2 shows that the extension from three-player race to $n$-player race $(n>3)$ does not essentially change our "reduction" result: once any two players win one victory each, all the remaining players are totally discouraged, and the continuation game reduces to a two-player race. This extreme discouragement becomes even more interesting if one takes

\footnotetext{
${ }^{5}$ It is worthwhile emphasizing that only players with no battle victories can become totally discouraged along the equilibrium path. Hence, it would
be extremely difficult to empirically detect total discouragement. Yet, we believe that almost any reader would be familiar with the phenomenon of a

${ }^{5}$ It is worthwhile emphasizing that only players with no battle victories can become totally discouraged along the equilibrium path. Hence, it would
be extremely difficult to empirically detect total discouragement. Yet, we believe that almost any reader would be familiar with the phenomenon of a researcher giving up on a research project after becoming aware of multiple working papers on precisely the same research question.
} 
into account the fact that the Tullock CSF we use provides stronger incentives (in comparison to an all-pay auction CSF) to lagging players and is known to make complete discouragement less likely (see, for instance, Karagözoğlu et al., 2017).

In the following remark, we present some comparative statics and limit results on the effect of the number of players on the equilibrium efforts, expected prizes, and rent dissipation.

Remark 1. As $n$ increases,

- the individual equilibrium effort at $T_{0}$ decreases (limit $=0$ ),

- the total equilibrium effort at $T_{0}$ increases (limit $=0.583 \mathrm{~V} / \mathrm{k}$ ),

- the individual expected prize as seen from $T_{0}$ decreases (limit $=0$ ),

- the total expected prize as seen from $T_{0}$ decreases (limit $=0$ ).

The complete rent dissipation mentioned above shares a similar flavor with the corresponding limit result in Cournot competition with variable number of (symmetric) firms (with constant marginal and average costs). In particular, as the number of firms goes to infinity, the equilibrium of Cournot competition converges to perfect competition, sweeping away economic profits; and here as the number of players goes to infinity, there is complete rent dissipation and positive expected prizes cease to exist.

In the following remark, we present comparative statics and limit results on the effect of the number of players on the momentum effect.

Remark 2. Assume that Player 1 wins the first period $T_{0}$, so that the game is at the node $T_{1}=(1,0, \ldots, 0)$. As $n$ increases,

- Player1's probability of winning period $T_{1}$ decreases, i.e. less pronounced momentum effect (limit $\left.=0.667\right)$,

- the ratio of Player1's probability of winning period $T_{1}$ to Player1's probability of winning period $T_{0}$ increases without bound.

\subsection{Extension to $n=3$ and $\mathrm{t}>2$}

Assume that $N=\{1,2,3\}$. Each player tries to win $t>2$ battles before any of the other players does. In our result, we provide a generalized solution for a generic node $T=\left(t_{1}, t_{2}, t_{3}\right)$, and accordingly, we avoid the number of wins when denoting the respective contest efforts unless there is a possibility of confusion. Moreover, we denote the expected prize of player $i$ at the node reached after player $j$ wins at this generic node by $V_{i j}$.

Proposition 3. Consider a generic node $T=\left(t_{1}, t_{2}, t_{3}\right)$ such that the expected prizes from all possible future states are known. Without loss of generality, assume that $t_{1} \geq t_{2} \geq t_{3}$. If $t_{1} \geq t_{2}=t_{3}$, then players exert

$$
e_{1}^{*}=\frac{x_{1}}{\left(x_{1}+x_{2}+x_{3}\right)^{2}}, \quad e_{2}^{*}=\frac{x_{2}}{\left(x_{1}+x_{2}+x_{3}\right)^{2}}, \quad e_{3}^{*}=\frac{x_{3}}{\left(x_{1}+x_{2}+x_{3}\right)^{2}}
$$

in the equilibrium, where

$$
x_{i}=\frac{\left(V_{i i}-V_{i j}\right)\left(V_{j j}-V_{j j^{\prime}}\right)+\left(V_{i i}-V_{i j^{\prime}}\right)\left(V_{j^{\prime} j^{\prime}}-V_{j^{\prime} j}\right)-\left(V_{j j}-V_{j j^{\prime}}\right)\left(V_{j^{\prime} j^{\prime}}-V_{j^{\prime} j}\right)}{\left(V_{i i}-V_{i j}\right)\left(V_{j j}-V_{j j^{\prime}}\right)\left(V_{j^{\prime} j^{\prime}}-V_{j^{\prime} i}\right)+\left(V_{i i}-V_{i j^{\prime}}\right)\left(V_{j j}-V_{j i}\right)\left(V_{j^{\prime} j^{\prime}}-V_{j^{\prime} j}\right)} k,
$$

for distinct players $i, j, j^{\prime} \in N$. If otherwise, Player 3 is totally discouraged, so that Players 1 and 2 exert

$$
e_{1}^{*}=\frac{x_{1}}{\left(x_{1}+x_{2}\right)^{2}} \quad \text { and } \quad e_{2}^{*}=\frac{x_{2}}{\left(x_{1}+x_{2}\right)^{2}}
$$

where

$$
x_{1}=\frac{k}{V_{22}-V_{21}} \text { and } x_{2}=\frac{k}{V_{11}-V_{12}} .
$$

Finally, for any type of generic node and each player $i \in N$ :

$$
E V_{i}^{t_{1}, t_{2}, t_{3}}=V_{i i}-k\left(2 e_{i}^{*}+\sum_{j \in N \backslash\{i\}} e_{j}^{*}\right) .
$$

Proof. See the Appendix.

Below, we present observations on rent dissipation and the momentum effect.

Rent dissipation: To begin with, there is an upper bound for the total rent dissipation: 0.7755 , and this is realized when $t=2$. The total rent dissipation converges to 0.7644 as $t$ increases. In fact, this value is reached as soon as $t=8$. The minimum rent dissipation occurs when $t=4$, and it is 0.7633 . This means that for a contest designer who wants to avoid rent dissipation as much as possible, $t=4$ is optimal.

Momentum effect: To see how the momentum effect responds to changes in $t$, assume that Player 1 wins the first period $T_{0}$. Then in the next period, $T_{1}=(1,0,0)$ : 
- If $t=2$, Player 1 's probability of winning $T_{1}$ is 0.714 ; his probability of winning the whole game (without losing a round) is $0.857(0.714)$.

- If $t=4$, Player 1 's probability of winning $T_{1}$ is 0.798 ; his probability of winning the whole game (without losing a round) is $0.898(0.781)$.

- If $t \geq 8$, Player 1 's probability of winning $T_{1}$ is 0.794 ; his probability of winning the whole game (without losing a round) is $0.896(0.777)$.

The winning probabilities when $t \in\{2, \ldots, 8\}$ do not necessarily behave in a monotonic fashion, but these observations indicate a more pronounced momentum effect for high values of $t$ (comparing $t=2$ with any $t \geq 8$ ). Furthermore, controlling for the intermediate values of $t$, we observe that $t=2$ always yields the minimum probabilities and that $t=4$ always yields the maximum probabilities.

\section{Concluding remarks}

Summary of the results: We show that the equilibrium of a multi-player game of race exhibits a severe discouragement effect and a partial violation of pervasiveness: as soon as any two players win one victory each, the game turns into a twoplayer race, as all of the other $n-2$ players drop out. This is different than the results in earlier works on two-player race, which show that each node is reached with some positive probability.

We also show that as long as the model is symmetric and the number of players is greater than two, the extreme discouragement effect is present independent of the number of players. Despite the fact that most players likely drop out in early stages of the game in equilibrium, the number of players still influences important markers of the contest. In particular, we show that keeping the victory threshold fixed, (i) an increase in the number of players increases the total rent dissipation and tempers the momentum effect, and (ii) as the number of players goes to infinity, all expected prizes are swept away. Moreover, keeping the number of players fixed, an increase in the victory threshold has, interestingly, a non-monotonic effect on rent dissipation and the momentum effect.

Extension to $n$-players and t-victories: When the number of players is $n>3$ and the victory threshold is $t>2$, we can show that there exists a unique subgame perfect Nash equilibrium in which any player who falls behind at least two other players is totally discouraged. For the existence result, consider first a node on which there are only two leading players. ${ }^{6}$ Take the strategy profile in which all non-leading players exert zero effort and the two leading players act as they would at the respective node in $\Gamma^{3, t}$ against a single discouraged player. We know that none of the leading or non-leading players would deviate from this strategy profile. Second, consider a node on which there are more than two leading players and derive another node such that the number of victories of all but two leading players are zero. Already knowing that all non-leading players are discouraged on the latter node, we can conclude that they would still be discouraged at the original node with more than two leading players. This concludes the existence part.

As for uniqueness, consider a node on which there are only two leading players. Consider an auxiliary contest played by the two leading players and an auxiliary player defined by the set of all non-leading players merged as one. Our equilibrium analysis for $\Gamma^{3, t}$ indicates that the auxiliary player is discouraged. Given the observation that being a non-leading player in the auxiliary contest is more advantageous than being one of the non-leading players in the original game, we can claim that all non-leading players would exert zero effort in the original contest. Moreover, if we consider a node on which there are more than two leading players, an analogous argument follows as in the existence part. This concludes the uniqueness part.

Possible extensions and future research: Future work may experimentally test the results reported in this paper, especially the results on the discouragement and momentum effects. Moreover, the current model can be extended by incorporating discounting, intermediate prizes, or losing penalties to investigate the respective changes in equilibrium behavior.

\section{Acknowledgments}

We would like to thank an anonymous reviewer for constructive comments that improved the paper. Emin Karagözoğlu thanks TÜBITAK (The Scientific and Technological Research Council of Turkey) for the post-doctoral research fellowship and the Department of Economics at Massachusetts Institute of Technology for their hospitality. The usual disclaimers apply.

\section{Appendix}

Proof of Proposition 1. The proof follows as a special case of the proof of Proposition 2. Hence it is omitted.

Proof of Proposition 2. At $T_{n} \equiv(1, \ldots, 1)$, each player has one victory. At this node, Player 1 maximizes

$$
p_{1}\left(e_{1}^{T_{n}}, \ldots, e_{n}^{T_{n}}\right) V-k e_{1}^{T_{n}}
$$

\footnotetext{
${ }^{6}$ Without loss of generality, assume that $t_{1} \geq t_{2} \geq \ldots \geq t_{n}$ where $t_{i}$ is the number of victories player $i$ has. Then player $i$ is a leading player if $t_{i} \geq t_{2}$.
} 
The first order condition with respect to $e_{1}^{T_{n}}$ is

$$
\frac{e_{2}^{T_{n}}+\ldots+e_{n}^{T_{n}}}{\left(e_{1}^{T_{n}}+\ldots+e_{n}^{T_{n}}\right)^{2}} V-k=0 .
$$

Considering the symmetric first order conditions for the other players, we see that $e_{1}^{T_{n} *}=\ldots=e_{n}^{T_{n} *}$ at the equilibrium. Then, we find that

$$
e_{1}^{T_{n} *}=\ldots=e_{n}^{T_{n} *}=\frac{(n-1) V}{n^{2} k} .
$$

This yields the following expected prizes:

$$
E V^{T_{n} *}=\left(\frac{V}{n^{2}}, \ldots, \frac{V}{n^{2}}\right) .
$$

At $T_{n-1} \equiv(1, \ldots, 1,0)$, Player 1 maximizes

$$
p_{1}\left(e_{1}^{T_{n-1}}, \ldots, e_{n}^{T_{n-1}}\right) V+p_{n}\left(e_{1}^{T_{n-1}}, \ldots, e_{n}^{T_{n-1}}\right) \frac{V}{n^{2}}-k e_{1}^{T_{n-1}} .
$$

The first order condition with respect to $e_{1}^{T_{n-1}}$ is

$$
\frac{e_{2}^{T_{n-1}}+\ldots+e_{n}^{T_{n-1}}}{\left(e_{1}^{T_{n-1}}+\ldots+e_{n}^{T_{n-1}}\right)^{2}} V-\frac{e_{n}^{T_{n-1}}}{\left(e_{1}^{T_{n-1}}+\ldots+e_{n}^{T_{n-1}}\right)^{2}} \frac{V}{n^{2}}-k=0 .
$$

Symmetrically, for every player $i=2, \ldots, n-1$, the respective first order condition is

$$
\frac{e_{1}^{T_{n-1}}+\ldots+e_{i-1}^{T_{n-1}}+e_{i+1}^{T_{n-1}}+\ldots+e_{n}^{T_{n-1}}}{\left(e_{1}^{T_{n-1}}+\ldots+e_{n}^{T_{n-1}}\right)^{2}} V-\frac{e_{n}^{T_{n-1}}}{\left(e_{1}^{T_{n-1}}+\ldots+e_{n}^{T_{n-1}}\right)^{2}} \frac{V}{n^{2}}-k=0 .
$$

We can see that $e_{1}^{T_{n-1} *}=\ldots=e_{n-1}^{T_{n-1} *}$ in equilibrium. Moreover, at this particular node, Player $n$ maximizes

$$
p_{n}\left(e_{1}^{T_{n-1}}, \ldots, e_{n}^{T_{n-1}}\right) \frac{V}{n^{2}}-k e_{n}^{T_{n-1}} .
$$

The first order condition with respect to $e_{n}^{T_{n-1}}$ is

$$
\frac{e_{1}^{T_{n-1}}+\ldots+e_{n-1}^{T_{n-1}}}{\left(e_{1}^{T_{n-1}}+\ldots+e_{n}^{T_{n-1}}\right)^{2}} \frac{V}{n^{2}}-k=0 .
$$

Utilizing $e_{1}^{T_{n-1 *}}=\ldots=e_{n-1}^{T_{n-1 *}}$ and solving the first order conditions with respect to $e_{1}^{T_{n-1}}$ and $e_{n}^{T_{n-1}}$ together, we obtain

$$
\frac{n-1}{n^{2}} e_{1}^{T_{n-1}} V=(n-2) e_{1}^{T_{n-1}} V+\frac{n^{2}-1}{n^{2}} e_{n}^{T_{n-1}} V
$$

This yields

$$
\left(3 n-n^{3}-1\right) e_{1}^{T_{n-1}}=\left(n^{2}-1\right) e_{n}^{T_{n-1}} .
$$

For $n \geq 3$, this condition cannot be satisfied by any $e_{1}^{T_{n-1}}, e_{n}^{T_{n-1}}>0$. Therefore, we conclude that $e_{n}^{T_{n-1} *}=0$ (i.e., Player $n$ quits the game at this node). This means that the node $(1, \ldots, 1)$ will never be reached in equilibrium. We, then, conclude that

$$
e_{1}^{T_{n-1} *}=\ldots=e_{n-1}^{T_{n-1} *}=\frac{n-2}{(n-1)^{2}} \frac{V}{k} .
$$

This yields the following expected prizes:

$$
E V^{T_{n-1} *}=\left(\frac{V}{(n-1)^{2}}, \ldots, \frac{V}{(n-1)^{2}}, 0\right) .
$$

Noting that the equilibrium analyses for every node at which $n-1$ players have one victory and one player has no victory follow symmetrically, we proceed to the analysis of the node $T_{n-2} \equiv(1, \ldots, 1,0,0)$ on which Player 1 maximizes

$$
p_{1}\left(e_{1}^{T_{n-2}}, \ldots, e_{n}^{T_{n-2}}\right) V+\left[p_{n-1}\left(e_{1}^{T_{n-2}}, \ldots, e_{n}^{T_{n-2}}\right)+p_{n}\left(e_{1}^{T_{n-2}}, \ldots, e_{n}^{T_{n-2}}\right)\right] \frac{V}{(n-1)^{2}}-k e_{1}^{T_{n-2}} .
$$

The first order condition with respect to $e_{1}^{T_{n-2}}$ is

$$
\frac{e_{2}^{T_{n-2}}+\ldots+e_{n}^{T_{n-2}}}{\left(e_{1}^{T_{n-2}}+\ldots+e_{n}^{T_{n-2}}\right)^{2}} V-\frac{e_{n+1}^{T_{n-2}}+e_{n}^{T_{n-2}}}{\left(e_{1}^{T_{n-2}}+\ldots+e_{n}^{T_{n-2}}\right)^{2}} \frac{V}{(n-1)^{2}}-k=0 .
$$


Symmetrically, for every player $i=2, \ldots, n-2$, it is

$$
\frac{e_{1}^{T_{n-2}}+\ldots+e_{i-1}^{T_{n-2}}+e_{i+1}^{T_{n-2}}+\ldots+e_{n}^{T_{n-2}}}{\left(e_{1}^{T_{n-2}}+\ldots+e_{n}^{T_{n-2}}\right)^{2}} V-\frac{e_{n+1}^{T_{n-2}}+e_{n}^{T_{n-2}}}{\left(e_{1}^{T_{n-2}}+\ldots+e_{n}^{T_{n-2}}\right)^{2}} \frac{V}{(n-1)^{2}}-k=0 .
$$

We can see that $e_{1}^{T_{n-2 *}}=\ldots=e_{n-2}^{T_{n-2 *}}$ in equilibrium. Moreover, at this particular node Player $n$ maximizes

$$
p_{n}\left(e_{1}^{T_{n-2}}, \ldots, e_{n}^{T_{n-2}}\right) \frac{V}{(n-1)^{2}}-k e_{n}^{T_{n-2}} .
$$

The first order condition with respect to $e_{n}^{T_{n-2}}$ is

$$
\frac{e_{1}^{T_{n-2}}+\ldots+e_{n-1}^{T_{n-2}}}{\left(e_{1}^{T_{n-2}}+\ldots+e_{n}^{T_{n-2}}\right)^{2}} \frac{V}{(n-1)^{2}}-k=0
$$

Symmetrically, for Player $n-1$, it is

$$
\frac{e_{1}^{T_{n-2}}+\ldots+e_{n-2}^{T_{n-2}}+e_{n}^{T_{n-2}}}{\left(e_{1}^{T_{n-2}}+\ldots+e_{n}^{T_{n-2}}\right)^{2}} \frac{V}{(n-1)^{2}}-k=0 .
$$

We can see that $e_{n-1}^{T_{n-2 *}}=e_{n}^{T_{n-2 *}}$. Utilizing $e_{n-1}^{T_{n-2 *}}=e_{n}^{T_{n-2 *}}$ and $e_{1}^{T_{n-2 *}}=\ldots=e_{n-2}^{T_{n-2 *}}$, and solving the first order conditions with respect to $e_{1}^{T_{n-2}}$ and $e_{n}^{T_{n-2}}$ together, we obtain

$$
\frac{n-2}{(n-1)^{2}} e_{1}^{T_{n-2}} V+\frac{1}{(n-1)^{2}} e_{n}^{T_{n-2}} V=(n-3) e_{1}^{T_{n-2}} V+\frac{2(n-1)^{2}-2}{(n-1)^{2}} e_{n}^{T_{n-2} V} .
$$

Rearranging terms yields

$$
(n-2) e_{1}^{T_{n-2}}+3 e_{n}^{T_{n-2}}=(n-1)^{2}(n-3) e_{1}^{T_{n-2}}+2(n-1)^{2} e_{n}^{T_{n-2}} .
$$

Since the coefficients on the RHS are always greater than the corresponding ones on the LHS, it must be that $e_{n}^{T_{n-2}}<0$. This implies that $e_{n-1}^{T_{n-2 *}}=e_{n}^{T_{n-2 *}}=0$ (i.e., Players $n-1$ and $n$ quit the game at this node). We, then, conclude that

$$
e_{1}^{T_{n-2 *}}=\ldots=e_{n-2}^{T_{n-2} *}=\frac{n-3}{(n-2)^{2}} \frac{V}{k} .
$$

This yields the following expected prizes:

$$
E V^{T_{n-2 *}}=\left(\frac{V}{(n-2)^{2}}, \ldots, \frac{V}{(n-2)^{2}}, 0,0\right) .
$$

Once again, we note that the equilibrium analyses for every node at which $n-2$ players have one victory each and two players have no victory follow symmetrically. Furthermore, at $T_{n-3} \equiv(1, \ldots, 1,0,0,0)$, following steps similar to the ones above yields that Players $n-2, n-1$, and $n$ are discouraged. On top of that, the equilibrium strategy for every player $i=1, \ldots, n-3$ is

$$
e_{i}^{T_{n-3 *}}=\frac{n-4}{(n-3)^{2}} \frac{V}{k}
$$

This yields the following expected prizes:

$$
E V^{T_{n-3 *}}=\left(\frac{V}{(n-3)^{2}}, \ldots, \frac{V}{(n-3)^{2}}, 0,0,0\right) .
$$

Similar results will carry over until the node $T_{1} \equiv(1,0, \ldots, 0)$. At this node Player 1 maximizes

$$
p_{1}\left(e_{1}^{T_{1}}, \ldots, e_{n}^{T_{1}}\right) V+\left[1-p_{1}\left(e_{1}^{T_{1}}, \ldots, e_{n}^{T_{1}}\right)\right] \frac{V}{4}-k e_{1}^{T_{1}} .
$$

The first order condition with respect to $e_{1}^{T_{1}}$ is

$$
\frac{e_{2}^{T_{1}}+\ldots+e_{n}^{T_{1}}}{\left(e_{1}^{T_{1}}+\ldots+e_{n}^{T_{1}}\right)^{2}} V-\frac{e_{2}^{T_{1}}+\ldots+e_{n}^{T_{1}}}{\left(e_{1}^{T_{1}}+\ldots+e_{n}^{T_{1}}\right)^{2}} \frac{V}{4}-k=0 .
$$

Moreover, Player 2 maximizes

$$
p_{2}\left(e_{1}^{T_{1}}, \ldots, e_{n}^{T_{1}}\right) \frac{V}{4}-k e_{2}^{T_{1}} .
$$


The first order condition with respect to $e_{2}^{T_{1}}$ is

$$
\frac{e_{1}^{T_{1}}+e_{3}^{T_{1}}+\ldots+e_{n}^{T_{1}}}{\left(e_{1}^{T_{1}}+\ldots+e_{n}^{T_{1}}\right)^{2}} \frac{V}{4}-k=0 .
$$

Symmetrically, for every player $i=3, \ldots, n$, it is

$$
\frac{e_{1}^{T_{1}}+\ldots+e_{i-1}^{T_{1}}+e_{i+1}^{T_{1}}+\ldots+e_{n}^{T_{1}}}{\left(e_{1}^{T_{1}}+\ldots+e_{n}^{T_{1}}\right)^{2}} \frac{V}{4}-k=0 .
$$

We can see that $e_{2}^{T_{1} *}=\ldots=e_{n}^{T_{1} *}$ in equilibrium. Utilizing this and solving the first order conditions with respect to $e_{1}^{T_{1}}$ and $e_{2}^{T_{1}}$, together, we obtain

$$
\left(e_{1}^{T_{1}}+(n-1) e_{2}^{T_{1}}\right) \frac{V}{4}=(n-1) e_{2}^{T_{1}} \frac{3 V}{4} .
$$

Accordingly, we have

$$
e_{1}^{T_{1} *}=(2 n-1) e_{2}^{T_{1} *} .
$$

Furthermore,

$$
e_{1}^{T_{1} *}=\frac{(2 n-1)(3 n-3)}{4(3 n-2)^{2}} \frac{V}{k}, e_{2}^{T_{1} *}=\ldots=e_{n}^{T_{1} *}=\frac{3 n-3}{4(3 n-2)^{2}} \frac{V}{k} .
$$

This yields the following expected prizes:

$$
E V^{T_{1} *}=\left(\frac{\left(21 n^{2}-24 n+7\right)}{4(3 n-2)^{2}} V, \frac{V}{4(3 n-2)^{2}}, \ldots, \frac{V}{4(3 n-2)^{2}}\right) .
$$

We now set

$$
V^{+} \equiv \frac{\left(21 n^{2}-24 n+7\right)}{4(3 n-2)^{2}} V \text { and } V^{-} \equiv \frac{V}{4(3 n-2)^{2}} .
$$

Finally, at $T_{0} \equiv(0, \ldots, 0)$ Player 1 maximizes

$$
p_{1}\left(e_{1}^{T_{0}}, \ldots, e_{n}^{T_{0}}\right) V^{+}+\left[1-p_{1}\left(e_{1}^{T_{0}}, \ldots, e_{n}^{T_{0}}\right)\right] V^{-}-k e_{1}^{T_{0}} .
$$

The first order condition with respect to $e_{1}^{T_{0}}$ :

$$
\frac{e_{2}^{T_{0}}+\ldots+e_{n}^{T_{0}}}{\left(e_{1}^{T_{0}}+\ldots+e_{n}^{T_{0}}\right)^{2}} V^{+}-\frac{e_{2}^{T_{0}}+\ldots+e_{n}^{T_{0}}}{\left(e_{1}^{T_{0}}+\ldots+e_{n}^{T_{0}}\right)^{2}} V^{-}-k=0 .
$$

Since we also have symmetric first order conditions for the other players, we should have $e_{1}^{T_{0} *}=\ldots=e_{n}^{T_{0} *}$ in equilibrium. Thus, we conclude that

$$
e_{1}^{T_{0} *}=\ldots=e_{n}^{T_{0} *}=\frac{(n-1)\left(21 n^{2}-24 n+6\right)}{4 n^{2}(3 n-2)^{2}} \frac{V}{k} .
$$

This yields the following expected prizes:

$$
E V^{T_{0} *}=\left(\frac{11 n^{2}-12 n+3}{2 n^{2}(3 n-2)^{2}} V, \ldots, \frac{11 n^{2}-12 n+3}{2 n^{2}(3 n-2)^{2}} V\right) .
$$

Proof of Proposition 3. Here we characterize the subgame perfect Nash equilibrium of $\Gamma^{3, t}$ in an iterated fashion. In step 1 , we start with a generic node $\left(t_{1}, t_{2}, t_{3}\right)$ and determine the conditions under which one player is totally discouraged. We identify three cases, in two of which, the laggard is totally discouraged. ${ }^{7}$ In step 2 , for each case, we characterize the equilibrium strategies at the generic node $T=\left(t_{1}, t_{2}, t_{3}\right)$ as functions of the equilibrium expected prizes from the possible states of the future. In step 3, writing the equilibrium expected prizes at $T$ in terms of the corresponding equilibrium strategies at $T$, we obtain an iterated structure which would reveal the subgame perfect Nash equilibrium for any $\Gamma^{3, t}$.

\footnotetext{
${ }^{7}$ For specific parts of this result, we utilize a software to solve for the equilibrium strategies (in terms of $k$ and $V$ ) for all players when $t \leq 20$. Following an observation that the expected prizes at the equilibrium converge as $t$ increases, we conjecture that our results would not change qualitatively in cases $t>20$.
} 
Step 1: Recall that we avoid the number of wins when denoting the respective contest efforts unless there is a possibility of confusion. We write that Player 1 maximizes

$$
\frac{e_{1}}{e_{1}+e_{2}+e_{3}} V_{11}+\frac{e_{2}}{e_{1}+e_{2}+e_{3}} V_{12}+\frac{e_{3}}{e_{1}+e_{2}+e_{3}} V_{13}-k e_{1}
$$

The first order condition with respect to $e_{1}$ is

$$
\frac{e_{2}+e_{3}}{\left(e_{1}+e_{2}+e_{3}\right)^{2}} V_{11}-\frac{e_{2}}{\left(e_{1}+e_{2}+e_{3}\right)^{2}} V_{12}-\frac{e_{3}}{\left(e_{1}+e_{2}+e_{3}\right)^{2}} V_{13}-k=0 \text {. }
$$

Letting

$$
x_{i} \equiv \frac{e_{i}}{\left(e_{1}+e_{2}+e_{3}\right)^{2}},
$$

the first order condition above becomes

$$
x_{2}\left(V_{11}-V_{12}\right)+x_{3}\left(V_{11}-V_{13}\right)=k .
$$

Considering the symmetric first order conditions for the other players, we can write the corresponding system of equations as

$$
\mathbf{V}_{i j} x_{i}=\left[\begin{array}{ccc}
0 & V_{11}-V_{12} & V_{11}-V_{13} \\
V_{22}-V_{21} & 0 & V_{22}-V_{23} \\
V_{33}-V_{31} & V_{33}-V_{32} & 0
\end{array}\right]\left[\begin{array}{l}
x_{1} \\
x_{2} \\
x_{3}
\end{array}\right]=\left[\begin{array}{l}
k \\
k \\
k
\end{array}\right]
$$

Solving for each $x_{i}$, we obtain

$$
\left[\begin{array}{l}
x_{1} \\
x_{2} \\
x_{3}
\end{array}\right]=\left[\begin{array}{ccc}
0 & V_{11}-V_{12} & V_{11}-V_{13} \\
V_{22}-V_{21} & 0 & V_{22}-V_{23} \\
V_{33}-V_{31} & V_{33}-V_{32} & 0
\end{array}\right]^{-1}\left[\begin{array}{l}
k \\
k \\
k
\end{array}\right] .
$$

Suppose that none of the players is discouraged at this node. Then, all non-diagonal entries of the $\mathbf{V}_{i j}$-matrix are positive and $x_{1}$ can be written as

$$
\frac{\left(V_{11}-V_{12}\right)\left(V_{22}-V_{23}\right)+\left(V_{11}-V_{13}\right)\left(V_{33}-V_{32}\right)-\left(V_{22}-V_{23}\right)\left(V_{33}-V_{32}\right)}{\left(V_{11}-V_{12}\right)\left(V_{22}-V_{23}\right)\left(V_{33}-V_{31}\right)+\left(V_{11}-V_{13}\right)\left(V_{22}-V_{21}\right)\left(V_{33}-V_{32}\right)} k
$$

We can write $x_{2}$ and $x_{3}$ in a symmetrical manner. If $x_{i}>0$ for $i \in N$, then $e_{i}>0$; however, if $x_{i} \leq 0$ for $i \in N$, then player $i$ would be discouraged. Accordingly, Player 1 is discouraged if and only if

$$
\frac{V_{11}-V_{12}}{V_{33}-V_{32}}+\frac{V_{11}-V_{13}}{V_{22}-V_{23}} \leq 1
$$

Also notice that there can be at most one discouraged player. From this point onwards, without loss of generality, we only consider the cases where Player 3 is discouraged, which occurs if and only if $x_{3} \leq 0$, i.e., if and only if

$$
\frac{V_{33}-V_{32}}{V_{11}-V_{12}}+\frac{V_{33}-V_{31}}{V_{22}-V_{21}} \leq 1 .
$$

We divide the following analysis into three cases:

Case 1: $\left(t^{\prime}, t^{\prime \prime}, t^{\prime \prime}\right)$ where $t^{\prime} \geq t^{\prime \prime}$. At this node, $V_{21}=V_{31}$ and $V_{22}=V_{33}$. Then

$$
\frac{V_{33}-V_{32}}{V_{11}-V_{12}}+\frac{V_{33}-V_{31}}{V_{22}-V_{21}}=1+\frac{V_{33}-V_{32}}{V_{11}-V_{12}}
$$

which is surely greater than 1 . We conclude that Player 3 is not discouraged.

Case 2: $\left(t^{\prime}, t^{\prime}, t^{\prime}-1\right)$. At this node, $V_{11}=V_{22}, V_{21}=V_{12}, V_{31}=V_{32}$, and $V_{13}=V_{23}=V_{33}$. Then

$$
\frac{V_{33}-V_{32}}{V_{11}-V_{12}}+\frac{V_{33}-V_{31}}{V_{22}-V_{21}}=\frac{2\left(V_{13}-V_{31}\right)}{V_{11}-V_{12}}
$$

which is to be compared to 1 in order to show whether Player 3 is discouraged or not. To further analyze this case, we refer to the following lemma.

Lemma 1. Let $t \leq 20$. Consider any node $\left(t^{\prime}, t^{\prime}, t^{\prime}-1\right)$. Then

- $V_{11}=V_{22}$ is bounded from below by $E V_{1}^{t-1, t-2, t-3}$,

- $V_{12}=V_{21}$ is bounded from above by $E V_{2}^{t-1, t-2, t-3}$, and

- $V_{13}=V_{23}=V_{33}$ is bounded from above by $E V_{1}^{t-1, t-1, t-1}$. 
where $E V_{i}^{t_{1}, t_{2}, t_{3}}$ denotes the equilibrium expected prize for player $i$ seen from the node $\left(t_{1}, t_{2}, t_{3}\right)$.

Proof. The proof is provided later.

The proof of Lemma 1 includes the equilibrium expected prizes at the nodes $(t-1, t-1, t-1)$ and $(t-1, t-2, t-3)$. Using these values, we calculate that

$$
\begin{aligned}
& 2\left(V_{13}-V_{31}\right) \leq 2 V_{13} \leq 2 E V_{1}^{t-1, t-1, t-1}=\frac{2 V}{9}, \\
& V_{11}-V_{12} \geq E V_{1}^{t-1, t-2, t-3}-E V_{2}^{t-1, t-2, t-3}=\frac{43 V}{64}-\frac{V}{64}=\frac{21 V}{32} .
\end{aligned}
$$

Accordingly, we conclude that Player 3 is discouraged at $\left(t^{\prime}, t^{\prime}, t^{\prime}-1\right)$, since

$$
\frac{2\left(V_{13}-V_{31}\right)}{V_{11}-V_{12}} \leq \frac{64}{189}<1 \text {. }
$$

It is worth emphasizing that Player 3 would also be discouraged at the node $\left(t^{\prime}, t^{\prime}, t^{\prime}-m\right)$ for any $m \in\left\{1, \ldots, t^{\prime}\right\}$.

Although Lemma 1 is provided for $t \leq 20$, observing how the expected prizes reported in the Appendix for $t=20$ converge, we conjecture that the results in Lemma 1 would hold also for $t>20 .^{8}$ The rest of the analysis would follow in a similar manner.

Case 3: $\left(t^{\prime}, t^{\prime \prime}, t^{\prime \prime \prime}\right)$ where $t^{\prime}>t^{\prime \prime}>t^{\prime \prime \prime}$. To show that Player 3 is discouraged, we directly refer to Case 2 . Starting from the node $\left(t^{\prime \prime}, t^{\prime \prime}, t^{\prime \prime \prime}\right)$, we can reach the former node only by $t^{\prime}-t^{\prime \prime}$ number of Player 1 victories. Then, it must be that

$$
E V_{3}^{t^{\prime}, t^{\prime \prime}, t^{\prime \prime \prime}} \leq E V_{3}^{t^{\prime \prime}, t^{\prime \prime}, t^{\prime \prime \prime}}
$$

Since $E V_{3}^{t^{\prime \prime}, t^{\prime \prime}, t^{\prime \prime \prime}}=0$, we conclude that Player 3 is discouraged at this node.

Step 2: Now that all possible situations are characterized in terms of discouragement, we are ready to characterize the equilibrium efforts in terms of the equilibrium expected prizes. In Case 1, none of the players is discouraged. Thus, as we have found earlier:

$$
x_{1}=\frac{\left(V_{11}-V_{12}\right)\left(V_{22}-V_{23}\right)+\left(V_{11}-V_{13}\right)\left(V_{33}-V_{32}\right)-\left(V_{22}-V_{23}\right)\left(V_{33}-V_{32}\right)}{\left(V_{11}-V_{12}\right)\left(V_{22}-V_{23}\right)\left(V_{33}-V_{31}\right)+\left(V_{11}-V_{13}\right)\left(V_{22}-V_{21}\right)\left(V_{33}-V_{32}\right)} k .
$$

We can write $x_{2}$ and $x_{3}$ in a symmetrical manner. The corresponding equilibrium efforts are

$$
e_{1}^{*}=\frac{x_{1}}{\left(x_{1}+x_{2}+x_{3}\right)^{2}}, \quad e_{2}^{*}=\frac{x_{2}}{\left(x_{1}+x_{2}+x_{3}\right)^{2}}, \quad e_{3}^{*}=\frac{x_{3}}{\left(x_{1}+x_{2}+x_{3}\right)^{2}} .
$$

In Cases 2 and 3, however, Player 3 is discouraged. So, we cannot use the same $x_{i}$ equations. We solve the same problem as if it is a two-player race. We can write the corresponding system of first order conditions as

$$
\left[\begin{array}{cc}
0 & V_{11}-V_{12} \\
V_{22}-V_{21} & 0
\end{array}\right]\left[\begin{array}{l}
x_{1} \\
x_{2}
\end{array}\right]=\left[\begin{array}{l}
k \\
k
\end{array}\right]
$$

From here we find that

$$
\left[\begin{array}{l}
x_{1} \\
x_{2}
\end{array}\right]=\left[\begin{array}{cc}
0 & V_{11}-V_{12} \\
V_{22}-V_{21} & 0
\end{array}\right]^{-1}\left[\begin{array}{l}
k \\
k
\end{array}\right]
$$

Knowing that all non-diagonal entries are positive:

$$
x_{1}=\frac{k}{V_{22}-V_{21}} \quad \text { and } \quad x_{2}=\frac{k}{V_{11}-V_{12}} .
$$

Similarly,

$$
e_{1}^{*}=\frac{x_{1}}{\left(x_{1}+x_{2}\right)^{2}} \quad \text { and } \quad e_{2}^{*}=\frac{x_{2}}{\left(x_{1}+x_{2}\right)^{2}} .
$$

This completes the characterization of the equilibrium efforts at any node in terms of the equilibrium expected prizes.

Step 3: We complete our analysis by characterizing the equilibrium expected prizes. Remember that at a generic node $\left(t_{1}, \overline{\left.t_{2}, t_{3}\right):}\right.$

$$
E V_{i}^{t_{1}, t_{2}, t_{3}}=\sum_{j \in\{1,2,3\}} \frac{e_{j}}{e_{1}+e_{2}+e_{3}} V_{i j}-k e_{i}
$$

\footnotetext{
${ }^{8}$ The analytical proof for this conjecture turns out to be far from trivial.
} 

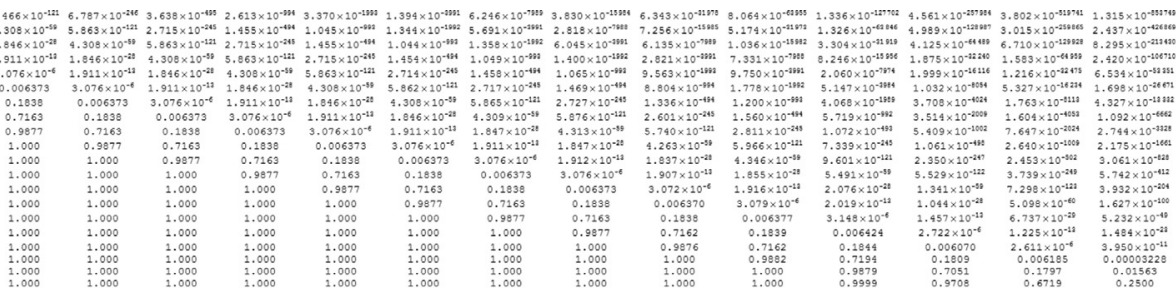

Fig. A1. Expected prizes for Player 1 when Player 3 has no victory.

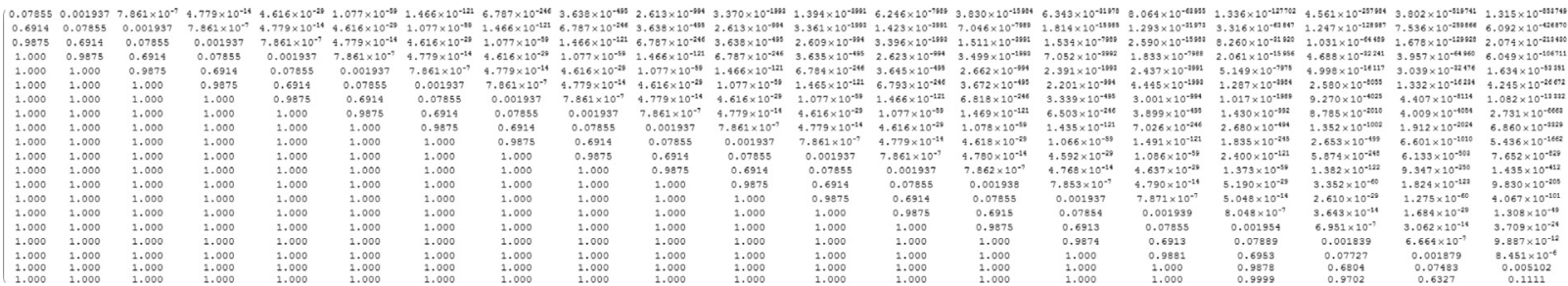

Fig. A2. Expected prizes for Player 1 when Player 3 has the same number of victories with the laggard among Players 1 and 2.

For any discouraged player $i$, we have $E V_{i}^{t_{1}, t_{2}, t_{3}}=0$. On the other hand, if player $i$ is not discouraged, then the first order condition with respect to $e_{i}$ holds. Consider the first order condition for Player 1 :

$$
\frac{e_{2}+e_{3}}{e_{1}+e_{2}+e_{3}} V_{11}-\frac{e_{2}}{e_{1}+e_{2}+e_{3}} V_{12}-\frac{e_{3}}{e_{1}+e_{2}+e_{3}} V_{13}=k\left(e_{1}+e_{2}+e_{3}\right) .
$$

In the LHS of this equation, adding and subtracting $e_{1} V_{11} /\left(e_{1}+e_{2}+e_{3}\right)$ yields

$$
V_{11}-\left(\frac{e_{1}}{e_{1}+e_{2}+e_{3}} V_{11}+\frac{e_{2}}{e_{1}+e_{2}+e_{3}} V_{12}+\frac{e_{3}}{e_{1}+e_{2}+e_{3}} V_{13}\right)=k\left(e_{1}+e_{2}+e_{3}\right),
$$

which eventually leads to

$$
V_{11}-\left(\sum_{j \in\{1,2,3\}} \frac{e_{j}}{e_{1}+e_{2}+e_{3}} V_{i j}-k e_{1}\right)=k\left(2 e_{1}+e_{2}+e_{3}\right) .
$$

It accordingly follows that

$$
E V_{1}^{t_{1}, t_{2}, t_{3}}=V_{11}-k\left(2 e_{1}^{*}+e_{2}^{*}+e_{3}^{*}\right) .
$$

The expected prizes for the other players are symmetric. This concludes the equilibrium analysis. ${ }^{9}$

Proof of Lemma 1. Below we provide two matrices for the expected prizes of Player 1 , when $t=20$. All entries are in the form of a scalar $a$ which indicates that Player1's expected prize is $a V$ at the respective node. In the first matrix (see Fig. A1), we restrict our analysis into cases in which Player 3 has no victory. The $(a, b)$-entry of the matrix is the expected prize at the node $(a-1, b-1,0)$. In the second matrix (see Fig. A2), we report the cases in which Player 3 has the same number of victories with the laggard among Players 1 and 2 . The $(a, b)$-entry of the matrix is the expected prize at the node $(a-1, b-1, \min \{a, b\}-1)$.

In Lemma 1 , we are interested in any node of the type $\left(t^{\prime}, t^{\prime}, t^{\prime}-1\right)$. We want to show that

- $V_{11}=V_{22}$ is bounded from below by $E V_{1}^{t-1, t-2, t-3}$,

- $V_{12}=V_{21}$ is bounded from above by $E V_{2}^{t-1, t-2, t-3}$, and

- $V_{13}=V_{23}=V_{33}$ is bounded from above by $E V_{1}^{t-1, t-1, t-1}$.

First, the expected prize $E V_{1}^{t-1, t-2, t-3}$ is in the $(20,19)-$ entry of the first matrix reported in Fig. A1: 0.6719. Moreover, the expected prize $V_{11}$ is in the $\left(t^{\prime}+2, t^{\prime}+1\right)$-entry of the same matrix. It can be seen that 0.6719 constitutes a lower bound for all values of $V_{11}$.

Second, the expected prize $E V_{2}^{t-1, t-2, t-3}$ is in the $(19,20)$-entry of the first matrix reported in Fig. A1: 0.0156. Moreover, the expected prize $V_{12}$ is in the $\left(t^{\prime}+1, t^{\prime}+2\right)$-entry of the same matrix. It can be seen that 0.0156 constitutes an upper bound for all values of $V_{12}$.

\footnotetext{
${ }^{9}$ Note that whether the continuation game after all but two players are discouraged is a symmetric or an asymmetric race depends on how many victories those two players won. If the first two victories were won by two different players, then the continuation game will be a symmetric race, otherwise it will be an asymmetric one.
} 
Third, the expected prize $E V_{1}^{t-1, t-1, t-1}$ is in the $(20,20)$-entry of the second matrix reported in Fig. A2: 0.1111. Moreover, the expected prize $V_{13}$ is in the $\left(t^{\prime}+1, t^{\prime}+1\right)$-entry of the same matrix. It can be seen that 0.1111 constitutes an upper bound for all values of $V_{13}$.

Notice that for any $t<20$, the corresponding expected prizes can be found in the reported matrices, since all possible cases in a race with a victory threshold of $t$ is embedded in a race with a victory threshold of $t+1$. This completes the proof.

Finally, it is worth noting that the expected prizes in the $\left(t^{\prime}+2, t^{\prime}+1\right)$ - and $\left(t^{\prime}+1, t^{\prime}+2\right)$-entry of the first matrix and the expected prizes in the $\left(t^{\prime}+1, t^{\prime}+1\right)$-entry of the second matrix converge as $t^{\prime} \in \mathbb{Z}_{+}$decreases. Moreover, the respective lower and upper bounds do not depend on the victory threshold $t$. Accordingly, we conjecture that the lemma would still hold for a victory threshold of $t>20$.

\section{References}

Baba, Y., 2012. A note on a comparison of simultaneous and sequential Colonel Blotto games. Peace Econ. Peace Sci. Public Policy 18.

Borel, E., 1921. La theore du jeu et les equations inte grales a noyau symetrique. Comptes Rendus de l'Acad. Sci. 173, 1304-1308. English translation by Savage. L., 1953. The theory of play and integral equations with skew symmetric kernels. Econometrica, 21, 97-100

Chowdhury, S.M., Kovenock, D., Sheremeta, R.M., 2013. An experimental investigation of Colonel Blotto games. Econ. Theory 52, 833-861.

Deck, C., Sheremeta, R.M., 2012. Fight or flight? Defending against sequential attacks in the game of siege. J. Confl. Resolut. 56, 1069-1088.

Duffy, J., Matros, A., 2017. Stochastic asymmetric Blotto games: an experimental study. J. Econ. Behav. Organiz. 139, 88-105.

Duverger, M., 1972. Factors in a Two-party and Multiparty System. Party Politics and Pressure Groups. Thomas Y. Crowell, New York.

Gelder, A., 2014. From custer to thermopylae: last stand behavior in multi-stage contests. Games Econ. Behav. 87, $442-466$.

Harris, C., Vickers, J., 1985. Perfect equilibrium in a model of race. Rev. Econ. Stud. 52, 193-209.

Harris, C., Vickers, J., 1987. Racing with uncertainty. Rev. Econ. Stud. 54, 1-21.

Hart, S., 2008. Discrete Colonel Blotto and General Lotto games. Int. J. Game Theory 36, 441-460.

Irfanoglu, B.Z., Mago, S.D., Sheremeta, R.M., 2015. New Hampshire effect: behavior in sequential and simultaneous election contests. MPRA Working Paper, No: 67520 .

Karagözoğlu, E., Sağlam, Ç., Turan, A., 2017. Tullock brings perseverance and suspense to tug-of-war. Bilkent University. Working Paper

Klumpp, T., Polborn, M.K., 2006. Primaries and the New Hampshire effect. J. Public Econ. 90, 1073-1114.

Konrad, K., 2012. Dynamic contests and the discouragement effect. Rev. Econ. Polit. 122, 233-256.

Konrad, K., Kovenock, D., 2009. Multi-battle contests. Games Econ. Behav. 66, 256-274.

Kvasov, D., 2007. Contests with limited resources. J. Econ. Theory 136, 738-748.

Mago, S.D., Sheremeta, R.M., 2017. Multi-battle contests: an experimental study. South. Econ. J. 84, 407-425.

Mago, S.D., Sheremeta, R.M., Yates, A., 2013. Best-of-three contest experiments: strategic versus psychological momentum. Int. J. Ind. Organiz. 31, 287-296.

Roberson, B., 2006. The Colonel Blotto game. Econ. Theory 29, 1-24.

Robson, A.R.W., 2005. Multi-item contests. Working Paper

Tullock, G., 1980. Efficient Rent Seeking. In: Buchanan, J.M., Tollison, R.D., Tullock, G. (Eds.), Toward a Theory of the Rent-Seeking Society. Texas A\&M University Press, pp. 97-112.

Vojnovic, M., 2016. Contest Theory: Incentive Mechanisms and Ranking Methods. Cambridge University Press, Cambridge. 